\title{
Statistical equilibrium dynamics
}

\author{
Michael Kiessling \\ Department of Mathematics, Rutgers University \\ Piscataway NJ 08854, USA \\ Version of Nov.29,2007
}

\begin{abstract}
We study the mean-field thermodynamic limit for a class of isolated Newtonian N-body systems whose Hamiltonian admits several additional integrals of motion. Examples are systems which are isomorphic to plasma models consisting of one specie of charged particles moving in a neutralizing uniform background charge. We find that in the limit of infinitely many particles the stationary ensemble measures with prescribed values of the integrals of motion are supported on the set of maximum entropy solutions of a (time-independent) nonlinear fixed point equation of mean-field type. Each maximum entropy solution of this fixed point equation can in turn be either a static or a stationary solution for the entropy-conserving Vlasov evolution, or even belong to a one-dimensional orbit of maximum entropy solutions which evolve into one another by the Vlasov dynamics. In short, the macrostates of individual members of an equilibrium ensemble are not necessarily themselves in a state of global statistical equilibrium in the strict sense. Yet they are always locally in thermodynamic equilibrium, and always global maximizers of the pertinent maximum entropy principle.
\end{abstract}

(C)2007 The author. This preprint may be reproduced for noncommercial purposes. (It is based on an invited talk delivered at the workshop "Dynamics and Thermodynamics of Systems with Long Range Interactions" in Assisi, Italy, 2007. An AIP layout of this preprint is going to appear in the AIP Conf. Proc. series.) 


\section{Introduction}

A short while ago I had an epiphany regarding equilibrium statistical mechanics. It happened while collaborating with Carlo Lancellotti on some curious Hamiltonian systems with unusual many body dynamics discovered by the Lynden-Bells [LBLB99, LBLB04]. It could (and should!) have happened much earlier, though it wouldn't have been an epiphany, then, if I had understood right from the beginning what equilibrium statistical mechanics is all about. In any case, until that moment of revelation I was under the erroneous yet I believe it's fair to say: common - impression that equilibrium statistical mechanics is entirely about the statistical microscopic foundations of the macroscopic concept of thermal equilibrium for Hamiltonian $N$-body systems when $N \gg 1$. Of course, equilibrium statistical mechanics is also about this, and even was invented specifically for this purpose, but that's not the whole story. The complete one is much richer and much more interesting.

In the following, I will first recall the "folklore" about Boltzmann's Bol96] ergodic ensemble measure, more familiar under the name Gibbs' Gib02 microcanonical ensemble measure

$$
\mathrm{d} \mu=\frac{1}{\Phi^{(N)}(E)} \delta(H-E) \mathrm{d}^{3 N} p \mathrm{~d}^{3 N} q
$$

where

$$
\Phi^{(N)}(E)=\int \delta(H-E) \mathrm{d}^{3 N} p \mathrm{~d}^{3 N} q
$$

is the normalizing factor. I will point out misconceptions in this folklore which become particularly troublesome when considering the restrictions of (1) to its stationary ergodic sub-ensembles associated with additional conserved quantities beside the Hamiltonian $H$. The true significance of (1) and its stationary sub-ensembles will become crystal clear once we pay due attention not just to the measures but also to the flow with respect to which they are stationary.

Throughout this presentation, with the exception of a few more general results it is always understood that the Hamiltonian $H$ of the classical $N$ body system under consideration is stable [i.e., collapse of parts of the system to a point is excluded] and confining [i.e., escape of particles from the system is excluded]. Prime examples of such systems with long range interactions are one-component Coulomb systems in a neutralizing background, which in particular includes the Lynden-Bells' system (a 4D Coulomb system restricted to $3 \mathrm{D}$ motions) but also 2D point vortex systems. Yet, for simplicity here I will only present a general discussion for $N$ body systems in 3D. The applications to the Coulomb systems will be published jointly with Carlo Lancellotti KiLa08.

Finally, to simplify notation I absorb normalizing factors such as (2) into the measures by writing $\underline{\delta}(\cdots)$ for normalized delta measures, and I write the sets on which the $\underline{\delta}^{\prime}$ s are supported as subscripts. 


\section{Folklore}

Without attempting to give a verbatim quotation from any particular book, I believe the following statement $(\mathrm{S})$ quite accurately captures the essence of similar statements in the pertinent literature:

(S) "An isolated classical $N$-body system with Hamiltonian $H$ and energy $E$ will spend most of its time in thermal equilibrium. In statistical mechanics, this equilibrium state is given by Boltzmann's stationary ergodic ensemble measure

$$
\mathrm{d} \mu=\underline{\delta}_{\{H=E\}}\left(\mathrm{d}^{3 N} p \mathrm{~d}^{3 N} q\right),
$$

unless additional constants of the motion exist; in this case there is a stationary probability measure for each ergodic component $\mathcal{M}$, given by

$$
\mathrm{d} \mu=\underline{\delta}_{\mathcal{M}}\left(\mathrm{d}^{3 N} p \mathrm{~d}^{3 N} q\right),
$$

where $\mathcal{M}=\cap_{k=0}^{K}\left\{I_{k}=C_{k}\right\}$, the $I_{k}$ are the isolating integrals, with $I_{0} \equiv H$, the $C_{k}$ their values, and $\mathrm{d}^{3 N} p \mathrm{~d}^{3 N} q$ stands for Liouville measure on phase space."

Since evaluating (3) in one of the usual ways (that is approximately, in most cases) will yield very satisfactory agreement with the empirical data almost anytime we inquire into the thermal equilibrium properties of a many body system which can be described classically, and since the general empirical relations of thermal equilibrium are recovered rigorously from (3) in some appropriate thermodynamic limit $N \rightarrow \infty$ dictated by the Hamiltonian, most of us, presumably, do not take any offense at reading statements such as (S), although we should. Indeed, in the line above (3), (S) makes the unfortunate suggestion that the macroscopic thermal equilibrium state of an individual physical system should be identified with a stationary probability measure over an ensemble of such systems, and this leads to some grossly incorrect conclusions in all but the most favorable circumstances.

What's more, $(\mathrm{S})$ is conceptually absurd. For instance, many invoke information theory to justify probability measures such as (3) and (4), becoming subjective, and then $(\mathrm{S})$ violates what should be enshrined as one of the 10 commandments of physics:

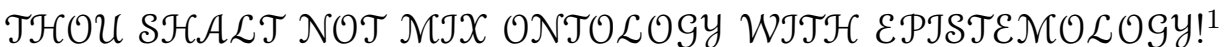

But even if the ensemble probabilities are interpreted in an objective manner the identification of the equilibrium macrostate of any individual physical system with a stationary ensemble probability measure is conceptually absurd, although in favorable cases when the overwhelming majority of systems in the

\footnotetext{
${ }^{1}$ Read: Do not confuse what a system does with what you can know about it! (The negation of this commandment is being worshiped in the orthodox quantum community, which seems exclusively concerned with observation and, most recently, information. But I don't want to take off on this tangent here. I have expressed my views in [Kie08.)
} 
ensemble have essentially the same macrostate then the results computed with this macrostate agree quantitatively with the results computed with the ensemble measure. Clearly, in such favorable cases some law of large numbers holds for the ensemble, i.e. if one formally let's $N \rightarrow \infty$ the empirical statistics of almost all members of the ensemble agrees with its ensemble mean. Boltzmann showed that such a law of large numbers holds for the perfect gas, assuming that $H$ is the only conserved quantity and using the ensemble measure (3). This prototype result by Boltzmann may have suggested the kind of thinking expressed in $(\mathrm{S})$; all the same, $(\mathrm{S})$ is absurd, and while masked by quantitative insignificance whenever a law of large numbers holds for the ensemble, the absurdity is there to stay and becomes manifest whenever the ensemble as a whole does not feature a law of large numbers - which is the case, for instance, at phase transitions of first order. Such phase transitions are defined quite sharply in the formalism of thermodynamics but not for (3) when $N<\infty$, yet the situation is quite easy to understand: if roughly fifty percent of the ensemble members have "spin +1 ," the other half have "spin -1 " (to use a simple representative example to illustrate the idea), the ensemble mean is "spin zero;" yet, each member of the ensemble has either spin +1 or spin -1 , not spin $\approx 0$.

Incidentally, Boltzmann was well aware of the fact that the notion of a phase transition and other thermodynamic notions as well are not unambigously defined in statistical mechanics for any $N<\infty$, and he also understood that all these thermodynamic notions can be sharply recovered from statistical mechanics in some limit $N \rightarrow \infty$. To rigorously implement this idea took a little longer, until Ruelle, Fisher, Lebowitz, Lieb, Thirring and others published their seminal papers on the subject in the $60 \mathrm{~s}$ and $70 \mathrm{~s}$ of the previous century, see [Rue69, Thi89]. The insight that the thermodynamic notions are not exact for $N<\infty$ yet rigorously recovered when $N \rightarrow \infty$ has lead to the suggestion that statements like $(S)$ have to be understood as implicitly referring to the (or some) "thermodynamic limit" $N=\infty$, with a system's macrostate being identified in general not with the limit of the measure (3) or (4) itself but with the extremal ergodic measures into which the limiting ensemble measure decompose.2 - the so-called pure states. This avoids the spin-up vs. spin-down problem of the above example (and all problems with similar dichotomies): the extremal ergodic measures are precisely the spin -1 and spin +1 measures. The pure states of the $N=\infty$ ensemble define ergodic subensembles; a law of large numbers holds for these sub-ensembles though not for the full ensemble. But has this now eliminated the conceptual problems?

Although the invocation of the limit $N \rightarrow \infty$ combined with the decomposition into extremal measures avoids the problems with dichotomies, the

\footnotetext{
${ }^{2}$ The set of probability measures on some measure space is convex, and every point in a convex set can be written as a convex linear combination of the extremal points of the set.
} 
proposal to define what is meant by a (macro)state of an individual $N$-body system (as distinct from computing it to a high degree of precision) in terms of the limit $N \rightarrow \infty$ was once commented on by Wigner thus 3 "[Name], you are a great man, but you are are not infinite." Wigner's point, translated into our setting, was that even granted the extremal ergodic measures of the $N=\infty$ ensemble were legitimate macrostates of the $N=\infty$ system (they are, as we shall see, though there is still a subtlety to be addressed), that still doesn't free us from the obligation to define a macrostate of a finite- $N$ system without invoking the limit $N \rightarrow \infty$. Of course, having found a sensible definition of macrostate for the $N=\infty$ system, a finite- $N$ definition should coincide with the $N=\infty$ definition in the limit. I will give a legitimate definition below.

I now address the second distinction of importance to which I have alerted you by highlighting in the paragraph after $(\mathrm{S})$ : the distinction between the equilibrium of an individual system and the stationarity of an ensemble measure. It is very easy to be confused about this distinction, even if you are not confused about the distinction between macrostate of an individual system and the state (i.e. probability measure) of an ensemble of such systems as discussed above. Part of the confusion is just a matter of semantics. With hindsight, Gibbs' choice of terminology, to call ensembles defined by (3) or (4) statistical equilibrium ensembles Gib02, while sensible, was not very fortunate. Another semantic item which contributes to the confusion is the fact that thermodynamic equilibrium of an individual $N$-body system has become synonymous with its statistical equilibrium, which terminology is based on the same notions which are behind Gibbs' terminology for a stationary ensemble of systems, only now applied to an individual system viewed as an ensemble of particles. The provocative title of my presentation is deliberately chosen to put you up against all the confusion right at the start: I insist, the title makes sense - but which sense? To find out we have now to confront the serious confusion, which is not at all a matter of semantics but belongs in a category described beautifully by Franz Kafka, who said "To correctly grasp an issue and to misunderstand the same [issue] do not exclude one another." (Translated from a quotation in [Rue69.)

A stationary ensemble measure by its very definition does not depend on time, and this of course remains so also when taking the limit $N \rightarrow \infty$. The limiting measure has a unique convex decomposition into extremal measures, and since the limiting measure is time-independent, so is its extremal decom-

\footnotetext{
${ }^{3}$ As told to me by Shelly Goldstein. Wigner commented on the suggestion by a distinguished seminar speaker that the measurement-induced collapse of the $n$-body wave function of the measured system, postulated in orthodox quantum mechanics, would perhaps result rigorously from the unitary Schrödinger evolution of the combined, $n+N$-body system in the limit $N \rightarrow \infty$, just as sharp phase transitions do. Clearly, the ghist of Wigner's remark is also valid in our context.
} 
position. If these extremal states represent the $N \rightarrow \infty$ limits of the possible macrostates of individual finite- $N$ systems in the ensemble, as we said earlier they would, only time-independent macrostates can be obtained.

Here then is something paradoxical: all the measures (4) are stationary, so what I just said applies to them. In particular, it applies to the special ensemble measure of the type (44) which is the next best known after (3), namely the one for $\mathcal{M}=\{H=E\} \cap\{J=L\}$ where the Hamiltonian $H$ and the $z$-component (say) $J$ of the angular momentum are conserved, having values $E$ and $L$, respectively. Now when $L \neq 0$ the system must rotate, in fact rigidly as Landau and Lifshitz will tell you. But we know that there are situations where the rotating system is not rotationally invariant, and then the macrostate of this system will be time-dependent, of the kind $\cos (\omega t-\varphi)$, with $\omega$ the constant angular frequency of the rigid rotation. For example, think of the triaxial Jacobi ellipsoids which describe rigidly rotating constant density self-gravitating fluids Cha69. How does this all fit together?

According to the folklore the paradox is resolved as follows: $\left(\mathrm{S}^{\prime}\right)$ "Since a stationary ensemble only yields stationary macrostates (read: equilibrium states), and since the rigidly rotating configurations with nonvanishing angular momentum rotate with a constant angular frequency, one has to apply the methods of equilibrium statistical mechanics in a co-rotating frame where the macrostate of the individual system is now stationary."

With regard to an inertial frame such rotating macrostates are usually called rotating equilibria. This prescription of how to apply the equilibrium statistical mechanics formalism leaves a strange taste in one's mouth, for it avoids the answer of the puzzle by circumventing it; yet the described procedure has worked fine since its inception, also for me.

After relegating the above paradox to the back of my mind for many years, it finally came full back into my conscience after Carlo Lancellotti and I had begun to work on the rigorous derivation of what the Lynden-Bells [LBLB99, LBLB04] had called a "perpetually pulsating equilibrium." They had discovered a curious Hamiltonian $N$-body system in three dimensional space with an unusual additional conservation law, besides the conventional ones for energy, momentum, and angular momentum. They managed to show, by studying the virial theorem, that the mean-square radial distance from the center of mass undergoes a sinusoidal motion for all $N$. They had also carried out some computer simulations with moderate $N$ and looked at the empirical statistics of velocities in a "co-pulsating frame," finding evidence for a Maxwellian - hence their terminology. But when Carlo and I set out to apply the conventional strategy advocated in the folklore ( $\left.\mathrm{S}^{\prime}\right)$, i.e. transforming the problem into a co-moving frame, I soon grew dissatisfied with this approach for conceptual reasons. The dynamics of the co-moving frame, while still simple, is already quite non-trivial because now the angular frequency 
of the rigid rotation is itself time-dependent (think of the familiar sight of an ice skater performing a pirouette, with her arms periodically extending from and retracting to her body). If we permit terms like "rotating equilibrium" and "pulsating equilibrium," we certainly have to also allow "rotatingpulsating equilibrium," as this is what the Lynden-Bells' "equilibrium" does in general. But what if someone discovers an even more curious Hamiltonian $N$-body system, with a "rotating-pulsating-twisting-bending-shearing-jerkingand-what-have-you equilibrium" — where does it stop? Are we not diluting the meaning of the word "equilibrium" until the construction becomes totally absurd?

Somehow the folklore was totally off target.

\section{$3 \quad$ Epiphany}

The crucial insight is that something everyone knows to be true anyhow for the finite- $N$ measures (44) in regard to the actual finite- $N$ microstates is true - by inheritance - also for the macrostates of the actual individual systems in the ensemble, and remains true in the limit $N \rightarrow \infty$.

In a nutshell, (4) lives on the $N$-body phase space, which consists of the generic phase points of $N$-body systems. Any point in $N$-body phase space defines a possible microstate of an $N$-body system. In addition, there is a Hamiltonian flow on this generic $N$-point phase space. Generic points do not move, they are independent variables. What evolves in time is the actual point in phase space which identifies the microstate of the actual $N$-body system. Its motion follows a particular flow line (if the Hamiltonian is time-independent) picked out by the initial actual phase point. So while (4) is stationary (with respect to the flow on $N$-body phase space), the ensemble of actual $N$-body systems it represents consists of systems whose actual phase points in general will not be stationary. Everyone knows that, and no one has a problem with that, even though some people may prefer to say the same thing differently.

Now, any mapping of the $N$-body microstates into $N$-body macrostates also induces a mapping of the flow on the space of generic microstates into a corresponding "flow" on the space of generic macrostates. Let's call this new "flow" the macroflow. Also the measure (44) will accordingly be mapped into a stationary measure on the space of generic macrostates. Let's call that induced ensemble measure the macrostate measure. If in the limit $N \rightarrow \infty$ the macrostate measure is supported only on such generic macrostates which are stagnation points for the limiting macroflow, then the limiting macrostates of the actual systems will be stationary too, and so the true finite- $N$ macrostates

will be approximately stationary, with small fluctuations. It is in the nature of macrostates that they don't care about most of what is going on at the microscopic level, so macrostates can be relatively quiet even if the underlying 
microstates evolve violently. But if that limiting measure is supported on generic macrostates at which the limiting macroflow is not stagnant, then the limiting macrostates of the actual systems will be dynamical, traversing an orbit in the subset of generic macrostates which form the support of the limiting stationary macrostate measure. By the same token as before, the true finite- $N$ macrostates will exhibit similar large scale macroscopic motions.

It should be perfectly clear by now that there is no conflict between the ensembles being stationary, implying that their extremal decompositions in the limit $N \rightarrow \infty$ are stationary as well, and the undeniable fact that the macrostates of individual actual systems making up the ensemble will in general exhibit macroscopic evolution in time.

In the remaining sections I will carefully explain how this general story pans out for the ensembles (4). I hope that my explanations are entirely correct, but I cannot rule out that I am still confused about this or that - think of Kafka! As appropriate for this conference, the general story is worked out for systems with long-range Galilei-invariant pair interaction which I will specify in more detail below; yet, the next section and the appendix are valid for Hamiltonians $H$ with more general Galilei invariant pair interactions. I will not invoke transformations into any co-moving non-inertial frames whatever; however, a Galilei boost into an inertial frame in which the center of mass is at rest is of a different category and will be performed (though the formalism does not even require that!). Of course, some limit $N \rightarrow \infty$ will be invoked, though not to define macrostates of the finite- $N$ systems but to calculate them approximately. Macrostates will be defined for finite- $N$ systems without invoking that limit. In fact, our first duty is to define what we mean by microscopic and macroscopic states, or micro- and macrostates for short. There is also an intermediate level frequently referred to as mesoscopic.

\section{Micro-, meso-, and macroscopic}

Taken literally the term "...-scopic" means something like "point of view," a very subjective notion, and we sure don't want to confuse what systems do with how we look at them! Fortunately there is a different way of putting it, namely as the objective structures produced by the $N$-body dynamics at the "finest scales," at "intermediate scales," and at "large scales." There is still some ambiguity as to what exactly is "fine," "intermediate," and "large," and this ambiguity will not go away! Yet at least the reference to subjective notions which seem implied by "scopic" is gone.

However, concepts must not be too arbitrary or they lose their significance. Since by "state" of a system one means a complete specification of the dynamical variables of the system, it must evolve (to good approximation) according to an autonomous dynamics at the scale under consideration; cf. GoLe04. 


\subsection{Microstates of individual systems}

Although the term microscopic scale does contain the ambiguity aluded to above, there fortunately is a clear cut choice for what the microscopic state of a Hamiltonian $N$-body system should be. Our Hamiltonian systems consist of $N \geq 2$ interacting nonrelativistic Newtonian point particles in Euclidean $\mathbb{E}^{3}$ with generic momenta $\boldsymbol{p}_{i} \in \mathbb{R}^{3}$ and positions $\boldsymbol{q}_{i} \in \mathbb{R}^{3}$ in an arbitrarily chosen inertial frame $\mathbb{R}^{3}$. Thus, for our Hamiltonian $N$-body systems then any point $\left(\boldsymbol{p}_{1}, \ldots, \boldsymbol{p}_{N}, \boldsymbol{q}_{1}, \ldots, \boldsymbol{q}_{N}\right)=X \in \mathbb{R}^{6 N}$ represents a possible state of the $N$-body system, or a generic state. If $\boldsymbol{q}_{i}(t)$ and $\boldsymbol{p}_{i}(t), i=1, \ldots, N$, are the $N$ particles' actual position and momentum vectors at time $t \in \mathbb{R}$ in an inertial frame, then $\left(\boldsymbol{p}_{1}(t), \ldots, \boldsymbol{q}_{N}(t)\right)=X_{t}$ is the actual state at time $t$. This is the most detailed characterization possible for the Hamiltonian $N$-body system, and therefore this is its microstate. This is the conventional definition. It is natural and unambiguous.

The disadvantage of this conventional definition of the microstate is that it is not well taylored to our needs. So instead we will work with a completely equivalent characterization of the microstate of a Hamiltonian $N$-body sytem, equivalent in the sense that there will be a one-to-one mapping between both types of objects. We identify each point $\left(\boldsymbol{p}_{1}, \ldots, \boldsymbol{q}_{N}\right)=X$ with a singular empirical "density" (a measure) on $\mathbb{R}^{6}$ as follows:

$$
\Delta_{X}(\boldsymbol{p}, \boldsymbol{q})=\sum_{i=1}^{N} \delta\left(\boldsymbol{p}-\boldsymbol{p}_{i}\right) \delta\left(\boldsymbol{q}-\boldsymbol{q}_{i}\right) .
$$

Obviously the map is bijective, i.e. $X \leftrightarrow \Delta_{X}$. Henceforth we will refer to (5) as the generic microstate of an $N$-body system. Note that (5) means physically the number density of particles at $q \in \mathbb{R}^{3}$ with momentum $p \in \mathbb{R}^{3}$. Since by $X_{t}$ we denote the actual point in phase space $\mathbb{R}^{6 N}$ at time $t$, then for us $\Delta_{X_{t}}(\boldsymbol{p}, \boldsymbol{q})$ is the actual microstate of the $N$-body system.

For the sake of completeness, we remark that this concept of microstate extends also to an $\infty$ many particles system. Intuitively: countably infinitely many points in $\mathbb{R}^{6}$ have Lebesgue measure 0 . Even though the empirical measure $\sum_{i=1}^{\infty} \delta\left(\boldsymbol{p}-\boldsymbol{p}_{i}\right) \delta\left(\boldsymbol{q}-\boldsymbol{q}_{i}\right)$ is not even locally finite if $\infty$ many points are densely distributed in some domain $\subset \mathbb{R}^{6}$, one can make sense out of it by looking at it as the collection of its finite $N$ truncations equipped with total variation $(T V)$ topology. In $T V$ topology then there will be no convergence to a continuum function when $N \rightarrow \infty$, so even though the $\infty$ many points may be dense in some domain, the topology is fine enough to distinguish the state from a continuum state. However, we will not need this construction. 


\subsection{Mesostates of individual systems}

We now face an irreducible ambiguity: what exactly constitutes a "mesoscopic scale"? There is no such thing as "the mesostate" of an $N$-body system per se. Yet in statistical physics one usually invokes this terminology in the context of kinetic theory, when a system is described in terms of some continuum formalism in $(\boldsymbol{p}, \boldsymbol{q})$ space $\mathbb{R}^{6}$; think of Boltzmann's transport equation for a dilute gas, or Vlasov's equations. Accordingly we could be tempted to call any such continuum density function $f(\boldsymbol{p}, \boldsymbol{q} ; t)$ of kinetic theory an "actual mesostate," and the time-independent generic functions the "generic mesostates." This is not quite adequate, though. The reason is that a continuum theory is of course even more detailed than an $N$-body theory, since $f$ needs to be specified at uncountably many points. This is hardly a description that considers only scales sufficiently bigger than the microscopic one of the previous subsection.

This inadequacy of the continuum description is ameliorated by restricting the (positive) continuum density functions $f(\boldsymbol{p}, \boldsymbol{q})$ in a suitable way. For instance one could work with piecewise linear, Lipschitz continuous functions with Lipschitz constant $<C$ for some $C$, and which linear pieces have their epicenters at a fixed "hypercubic" lattice. This would be quite adequate.

To make our life easier we are a little more cavalier and allow functions $f(\boldsymbol{p}, \boldsymbol{q})$ which are uniformly Lipschitz continuous and uniformly iterated Lipschitz continuous. Basically this says that such functions cannot be too steep, and cannot oscillate on too small scales; and since they are non-negative and need to integrate to a finite number, they cannot develop singularities when $(\boldsymbol{p}, \boldsymbol{q})$ go to infinity. We denote this class of functions $\mathscr{M} \mathscr{E}$. It is understood that some uniform bound on the Lipschitz and iterated Lipschitz constants is chosen; they clearly restrict the possibilities, but how exactly is a matter of choice and good judgement and part of the irreducible ambiguity of any notion such as mesoscopic, or for that matter also of macroscopic.

Next we associate to each microstate $\Delta_{X}$ a mesostate $f_{X}$. This map may be but need not be bijective. To simplify the discussion, we work with the normalized empirical densities $\Delta_{X}=N^{-1} \Delta_{X}$ and understand henceforth that any $f_{X}$ is normalized such as to integrate to 1 as well. We now pick a suitable Kantorovich-Rubinstein metric $d_{\mathrm{KR}}$ (which metrices weak convergence of normalized measures) and define $f_{X}$ as the minimizer of the KR distance between $\underline{\Delta}_{X}$ and $\mathscr{M} \mathscr{E}$, i.e.

$$
d_{\mathrm{KR}}\left(\underline{\Delta}_{X}, f_{X}\right)=\min \left\{d_{\mathrm{KR}}\left(\underline{\Delta}_{X}, f\right) \mid f \in \mathscr{M} \mathscr{E}\right\}
$$

if the minimum exists; if it doesn't, since the infimum of the set on the r.h.s. is certainly strictly positive, one can stipulate some convenient rule to choose an $f_{X}$ among those $f$ which differ from $\Delta_{X}$ in KR distance by not more than the infimum plus $\epsilon$. If that sounds somewhat ambiguous, that's because it is, but 
such ambiguities are acceptable given the inherent ambiguity in the notion of a "mesoscopic scale."

\subsection{Macrostates of individual systems}

Of course, the same kind of irreducible ambiguity that haunts the "mesoscopic scale" also raises the question of what exactly constitutes a "macroscopic scale;" and again there is no objective answer to this. In the statistical physics literature, the main distinction between the "macroscale" and the "mesoscale" is that in the former one now does not resolve the variations in $\boldsymbol{p}$ space. More to the point, a macrostate is usually the small collection of fluid dynamical functions on $\boldsymbol{q}$ space $\mathbb{R}^{3}$, like mass density, velocity field, and kinetic energy density field or pressure field. Such functions can be obtained from the mesostates by taking the first few moments over $\boldsymbol{p}$ space.

\section{Finite $N$}

\subsection{Dynamics}

The dynamics of each system is governed by a Hamiltonian of the type

$$
H^{(N)}\left(\boldsymbol{p}_{1}, \boldsymbol{q}_{1} ; \ldots ; \boldsymbol{p}_{N}, \boldsymbol{q}_{N}\right):=\sum_{1 \leq i \leq N} \frac{1}{2 m_{i}}\left|\boldsymbol{p}_{i}\right|^{2}+\sum_{1 \leq i<j \leq N} \sum_{i} e_{i} e_{j} V\left(\left|\boldsymbol{q}_{i}-\boldsymbol{q}_{j}\right|\right)
$$

where the $m_{i}>0$ are the inertial masses and the $e_{i} \in \mathbb{R}$ "generalized charges." The pair interaction potential $V(r)$ will be of long range and locally integrable to admit a mean-field limit when $N \rightarrow \infty$, but many facts do not depend on all the details of $V$. In particular, the following familiar conservation laws hold for a larger class of $V$ than just those allowing mean-field limits. Since we need the conservation laws to set up our ensembles, we collect them here.

Lemma 1. Assume the pair interaction potential $V$ satisfies $V \in \mathfrak{C}^{2}\left(\mathbb{R}_{+}, \mathbb{R}\right)$, guaranteeing local existence and uniqueness of the dynamics. Then by the invariance of $H^{(N)}$ under time and space translations, as well as space rotations, the isolating integrals of motion are: the Hamiltonian $H^{(N)}$ given in (7), the total momentum phase space function

$$
\boldsymbol{P}^{(N)}\left(\boldsymbol{p}_{1}, \boldsymbol{q}_{1} ; \ldots ; \boldsymbol{p}_{N}, \boldsymbol{q}_{N}\right):=\sum_{1 \leq i \leq N} \boldsymbol{p}_{i},
$$

and the total angular momentum phase space function

$$
\boldsymbol{J}^{(N)}\left(\boldsymbol{p}_{1}, \boldsymbol{q}_{1} ; \ldots ; \boldsymbol{p}_{N}, \boldsymbol{q}_{N}\right):=\sum_{1 \leq i \leq N} \boldsymbol{q}_{i} \times \boldsymbol{p}_{i} .
$$


Lemma 1 exhausts the isolating integrals of motion w.r.t. the center-ofmass frames which are shared by all Hamiltonian systems characterized by (7) under the stated hypotheses on $V$. Additional isolating integrals for all $N \geq 2$ may exist for special cases of $V$, and we shall encounter an example later on.

Moreover, on account of $H^{(N)}$ changing only by an additive constant under a Galilei boost, what is sometimes called the system's centroid and given by $\frac{1}{M} \sum_{1 \leq i \leq N}\left(m_{i} \boldsymbol{q}_{i}(t)-t \boldsymbol{p}_{i}(t)\right)$, is conserved, too; here $M=\sum_{1 \leq i \leq N} m_{i}$ is the total mass of the system, which is independent of the choice of inertial frame. However, the centroid is not the evaluation of a $t$-independent function on $\left(\boldsymbol{p}_{1}, \ldots, \boldsymbol{q}_{N}\right)$ space and therefore not an isolating integral. Yet, since by the Galilean invariance of Newtonian point mechanics any particular one of our $N$-body systems can always be described w.r.t. any of its center-ofmass frames (any co-moving inertial frame for which the center of mass is the origin of that frame), without loss of generality it suffices to study $N$-body systems under the holonomic scleronomous constraints $\boldsymbol{P}^{(N)}\left(\boldsymbol{p}_{1}, \ldots, \boldsymbol{q}_{N}\right)=\mathbf{0}$ and $\boldsymbol{C}^{(N)}\left(\boldsymbol{p}_{1}, \boldsymbol{q}_{1} ; \ldots ; \boldsymbol{p}_{N}, \boldsymbol{q}_{N}\right)=\mathbf{0}$, where

$$
\boldsymbol{C}^{(N)}\left(\boldsymbol{p}_{1}, \boldsymbol{q}_{1} ; \ldots ; \boldsymbol{p}_{N}, \boldsymbol{q}_{N}\right):=\frac{1}{M} \sum_{1 \leq i \leq N} m_{i} \boldsymbol{q}_{i}
$$

is the center-of-mass phase space function. As far as the stationary ergodic subensembles go that we introduce below, these center-of-mass-frame constraints can be treated on the same footing as the the true isolating integrals.

Hence, to unify the notation, we include both the true isolating integrals and the scleronomic constraint functions in a list of functions $I_{k}, k=0,1, \ldots$, simply called invariants, with $C_{k}$ denoting the values they take. In particular, we define: $I_{0}:=H^{(N)}$ with $C_{0}=E,\left(I_{1}, I_{2}, I_{3}\right):=\boldsymbol{P}^{(N)}$ with $\left(C_{1}, C_{2}, C_{3}\right):=$ $\mathbf{0},\left(I_{4}, I_{5}, I_{6}\right):=\boldsymbol{J}^{(N)}$ with $\left(C_{4}, C_{5}, C_{6}\right)=\boldsymbol{L}$, and $\left(I_{7}, I_{8}, I_{9}\right):=\boldsymbol{C}^{(N)}$ with $\left(C_{7}, C_{8}, C_{9}\right)=\mathbf{0}$, where $E \in \mathbb{R}$ and $\boldsymbol{L} \in \mathbb{R}^{3}$ are time-independent parameters.

Incidentally, the phase space functions (7), (8), (9), (10) are not all in involution; the invariants $H^{(N)}, \boldsymbol{P}^{(N)},\left|\boldsymbol{J}^{(N)}\right|^{2}, \boldsymbol{J}^{(N)} \cdot \boldsymbol{e}$ are, where $\boldsymbol{e}$ is any fixed unit vector in space. Moreover, in any of our center-of-mass frames $\boldsymbol{C}^{(N)}$ is in involution with $H^{(N)}$ but not with $\boldsymbol{P}^{(N)}$ and $\boldsymbol{J}^{(N)}$. This is not a problem, for we will characterise the ergodic submanifolds conveniently in such a way that we do not need to find a set of invariants in involution; however, it is implicitly understood in the following that any additional invariants $I_{k}$ with $k=10, \ldots$ will not be redundant.

Lemma 2. Let $K+1(\geq 10)$ be the number of invariants admitted by $H^{(N)}$, with $V$ as in Lemma 1.

a) Let $\mathbb{M}_{K}$ denote the manifold

$$
\mathbb{M}_{K} \equiv\left\{\left(\boldsymbol{p}_{1}, \ldots, \boldsymbol{q}_{N}\right) \in \mathbb{R}^{6 N} \mid I_{k}=C_{k}, k=0, \ldots, K\right\} .
$$


Then $\mathbb{M}_{K}$ is invariant under the flow generated by the Hamiltonian $H^{(N)}$.

b) Let $\mu^{(N)}$ denote the singular measure supported on $\mathbb{M}_{K}$ given by

$$
\mathrm{d} \mu^{(N)}=\delta_{\cap_{k=0}^{K}\left\{I_{k}=C_{k}\right\}}\left(\mathrm{d}^{3 N} p \mathrm{~d}^{3 N} q\right) .
$$

Then (12) is invariant under the adjoint flow associated with $H^{(N)}$, i.e. (12) is a stationary weak solution of Liouville's equation

$$
\partial_{t} \mu+\left\{\mu, H^{(N)}\right\}=0 .
$$

So far, the conditions for Lemmas 1 and 2 also cover Hamiltonian systems of type (17) with physically relevant pair interactions, such as $V(x)=K / x$ with $e_{i} \in \mathbb{R}$ (Coulombian electricity) and $V(x)=-K / x$ with $e_{i} \in \mathbb{R}_{+}$(Newtonian gravity), as well as $V(x)=K\left(x^{-12}-x^{-6}\right)$ with $e_{i} \in \mathbb{R}_{+}$(Lennard-Jones molecular pair potential). Unfortunately, neither of these Hamiltonian systems is self-confining, and the jury is still out on the question of the generic global existence of the dynamics in the gravitational and electrical $N$-body problems. We now impose stronger conditions on $V$ which guarantee global existence of the dynamics and self-confinement of the system of particles.

Proposition 1. Let $K+1(\geq 10)$ be the number of invariants admitted by $H^{(N)}$, with $V$ satisfying the hypotheses in Lemma 1.

a) If in fact $V \in \mathfrak{C}^{2}\left(\mathbb{R}_{+}, \mathbb{R}_{+}\right)$with $-e_{i} e_{j} V^{\prime}(r) \stackrel{r \downarrow 0}{\longrightarrow} \infty$ for all $i, j$, or $V \in$ $\mathfrak{C}^{2}\left(\overline{\mathbb{R}}_{+}, \mathbb{R}_{+}\right)$with $V^{\prime}(r) \stackrel{r \downarrow 0}{\longrightarrow} 0$, and in either case $\lim \sup _{r \rightarrow \infty}\left|V^{\prime}(r)\right| / r \leq C$, then the dynamics exists globally in time.

b) If in addition to the hypotheses in a) we have $\lim _{r \rightarrow \infty} e_{i} e_{j} V(r)=+\infty$ for all pairs $i, j$, then (12) is a finite measure.

Henceforth we assume that $V$ satisfies the hypotheses of Proposition 1b). Note that when $N>2$ this implies that either $e_{i}>0 \forall i$ or $e_{i}<0 \forall i$.

\section{$5.2 \quad$ Statistics}

In the section "Folklore" I spoke several times of "a law of large numbers" as $N \rightarrow \infty$. This is related to, but not to be confused with, a familiar law of large numbers which holds (under mild conditions) for ensembles of i.i.d. finite- $N($ !) systems.

Denoting normalized (12) by $\underline{\mu}^{(N)}$, i.e. $\int \mathrm{d} \underline{\mu}^{(N)}=1$, this can be interpreted as the single-system a-priori probability measure for a Newtonian $N$-body system in phase space $\mathbb{R}^{6 N}$ to be at $X$ if all that is given about the system is its Hamiltonian $H^{(N)}$ with energy $E$, its total angular momentum $\boldsymbol{L}$, its center of mass $=\mathbf{0}$, its total momentum $=\mathbf{0}$, and the values $C_{k}$ for whatever other invariants there are; with $H^{(N)}$ also the number $N$ of particles and total mass 
$M$ are given. Thus, if $\mathscr{B}$ denotes the Borel sets of $\mathbb{R}^{6 N}$, then $\left(\mathbb{R}^{6 N}, \mathscr{B}, \mu^{(N)}\right)$ is our single-system probability space. The conventional microstate of a single system is now a random vector $X=\left(\mathcal{P}_{1}, \ldots, \mathcal{P}_{N}, Q_{1}, \ldots, Q_{N}\right) \in \mathbb{R}^{3 N} \times \mathbb{R}^{3 N}$, the specification of which amounts to giving exactly the positions and momenta of each particle in the $N$-body system. If $B \subset \mathbb{R}^{6 N}$ is a Borel set, then the probability of $X$ being in $B$ is

$$
\operatorname{Prob}(X \in B)=\underline{\mu}^{(N)}(B) .
$$

Clearly, Prob $(X \in B)=0$ unless $B \cap \mathbb{M}_{K} \neq \emptyset$.

The Boltzmann ergodic subensemble associated with (12) is an infinite family of i.i.d. random vectors $X^{(j)}$, each with a-priori distribution (12), and each one of which represents a Newtonian $N$-body system which is governed by the same Hamiltonian $H^{(N)}$ and has the same energy $E$, center of mass $=\mathbf{0}$, total momentum $=\mathbf{0}$, total angular momentum $\boldsymbol{L}$, and same values $C_{k}$ for whatever other invariants there are; of course, each system also has the same number $N$ of particles and total mass $M$. Any ordered set of $\ell$ copies of $X$ is a random vector $\left(X^{(1)}, \ldots, X^{(\ell)}\right) \in \mathbb{R}^{\ell 6 N}$ with joint distribution $\underline{\mu}^{(N) \times \ell}$. For this family of i.i.d. systems the conventional weak law of large numbers (WLLN) holds.

We are going to state the WLLN in the form adapted to our discussion of the concept of a system's "microstate" as $\Delta_{X}(\boldsymbol{p}, \boldsymbol{q})$. Accordingly, $X$ is identified with a singular-empirical-density-valued random variable $\Delta_{x}(\boldsymbol{p}, \boldsymbol{q})$, and the measure (12) is identified with a single-system a-priori probability measure $\tilde{\mu}^{(N)}$ on the convex set of all singular densities (i.e. measures) on $\mathbb{R}^{6}$. Clearly, $\tilde{\mu}^{(N)}$ is supported only on the set of all singular densities of the type $\Delta_{X}(\boldsymbol{p}, \boldsymbol{q})$. Boltzmann's ergodic ensemble is therefore identical to an infinite family of i.i.d. random densities $\Delta_{x}(\boldsymbol{p}, \boldsymbol{q})$, each with a-priori distribution $\tilde{\mu}^{(N)}$. By convexity, the empirical mean of a size- $\ell$ sample of i.i.d. empirical densities,

$$
\bar{\Delta}_{X}^{(\ell)}(\boldsymbol{p}, \boldsymbol{q})=\frac{1}{\ell} \sum_{j=1}^{\ell} \Delta_{X^{(j)}}(\boldsymbol{p}, \boldsymbol{q})
$$

is again a density of the same total measure $=N$. For the normalized measure $\underline{\mu}^{(N)}$ defined by (12), denote by $\underline{\mu}_{1, i}^{(N)}\left(\mathrm{d}^{3} p \mathrm{~d}^{3} q\right)$ its first marginal measure for the $i$-th particle variables $\left(\boldsymbol{p}_{i}, \boldsymbol{q}_{i}\right)$, evaluated at $(\boldsymbol{p}, \boldsymbol{q})$. Recall (see, e.g. Dur96, Dud02]) that a family of probability measures $\nu_{n}$ on $\mathbb{R}^{d}$ is said to converge weakly to $\nu$ if $\int g(x) \mathrm{d} \nu_{n} \rightarrow \int g(x) \mathrm{d} \nu$ for all bounded continuous functions $g \in \mathfrak{C}_{b}^{0}\left(\mathbb{R}^{d}\right)$. Recall furthermore (e.g. [Dud02]) that weak convergence is metrized by some Kantorovich-Rubinstein metric $d_{\mathrm{KR}}$. We are now ready to state the weak law of large numbers. 
Theorem 1. For Boltzmann's ergodic ensemble of i.i.d. random vectors $X$, each with a-priori distribution (12), we have the weak law of large numbers,

$$
\lim _{\ell \rightarrow \infty} \operatorname{Prob}\left(d_{\mathrm{KR}}\left(\bar{\Delta}_{x}^{(\ell)}(\boldsymbol{p}, \boldsymbol{q}) \mathrm{d}^{3} p \mathrm{~d}^{3} q, \sum_{i=1}^{N} \underline{\mu}_{1, i}^{(N)}\left(\mathrm{d}^{3} p \mathrm{~d}^{3} q\right)\right)>\epsilon\right)=0 .
$$

We follow the probabilists custom and rephrase the WLLN shorter thus:

$$
\bar{\Delta}_{X}^{(\ell)}(\boldsymbol{p}, \boldsymbol{q}) \mathrm{d}^{3} p \mathrm{~d}^{3} q \stackrel{\ell \rightarrow \infty}{\longrightarrow} \sum_{i=1}^{N} \underline{\mu}_{1, i}\left(\mathrm{~d}^{3} p \mathrm{~d}^{3} q\right) \quad \text { in } \quad \text { probability. }
$$

So far all the particles in an individual $N$-body system may have different masses and charges 4 Many systems of interest in nature do consist of only a handful of species, and the prototype systems to study are one-specie systems with $m_{i}=m>0$ and $e_{i}=e \in \mathbb{R}$ for all $i$ in (7), so the Hamiltonian becomes

$$
H^{(N)}\left(\boldsymbol{p}_{1}, \ldots, \boldsymbol{q}_{N}\right)=\sum_{1 \leq i \leq N} \frac{1}{2 m}\left|\boldsymbol{p}_{i}\right|^{2}+\sum_{1 \leq i<j \leq N} \sum^{2} V\left(\left|\boldsymbol{q}_{i}-\boldsymbol{q}_{j}\right|\right) .
$$

For such single specie systems the WLLN simplifies to the following.

Theorem 2. For the Hamiltonian (18), all the first marginal measures are identical due to the permutation invariance of $H^{(N)}$ which is inherited by the product over all the isolating integrals in (12). Thus, $\underline{\mu}_{1, i}^{(N)}\left(\mathrm{d}^{3} p \mathrm{~d}^{3} q\right)=$ $\underline{\mu}_{1}^{(N)}\left(\mathrm{d}^{3} p \mathrm{~d}^{3} q\right)$ for all $i=1, \ldots, N$. Accordingly, as $\ell \rightarrow \infty$,

$$
\bar{\Delta}_{X}^{(\ell)}(\boldsymbol{p}, \boldsymbol{q}) \mathrm{d}^{3} p \mathrm{~d}^{3} q \longrightarrow N \underline{\mu}_{1}^{(N)}\left(\mathrm{d}^{3} p \mathrm{~d}^{3} q\right)
$$

in probability.

Note that no limit $N \rightarrow \infty$ is involved at this level.

\section{The limit $N \rightarrow \infty$}

From now on our Hamiltonian will be (18). In addition, we are interested now only in those Hamiltonians (18) which permit a mean-field continuum limit $N \rightarrow \infty$. Therefore, in addition to the hypotheses of Proposition 1b, from now on we assume also that $V$ satisfies $V \circ|.| \in \mathfrak{L}_{\text {loc }}^{1}\left(\mathbb{R}^{3}\right)$, and we also ask that $\lim _{r \rightarrow \infty} V(r) / r=\infty$.

\footnotetext{
${ }^{4}$ Physical examples of large $N$-body systems with a distribution of particle masses are stellar clusters.
} 


\subsection{Dynamics of microstates}

Having identified points $X$ on $\mathbb{M}_{K} \subset \mathbb{R}^{6 N}$ with empirical densities $\Delta_{X}(\boldsymbol{p}, \boldsymbol{q})$, we note that the Hamiltonian flow on $\mathbb{M}_{K}$ which transports any point $X_{0}$ on $\mathbb{M}_{K}$ at time 0 to a corresponding point $X_{t}$ on $\mathbb{M}_{K}$ at time $t$ also defines the dynamics of the empirical densities $\Delta_{X}(\boldsymbol{p}, \boldsymbol{q})$; namely, the initial empirical density $\Delta_{X_{0}}(\boldsymbol{p}, \boldsymbol{q})$ evolves into $\Delta_{X_{t}}(\boldsymbol{p}, \boldsymbol{q})$ at time $t$. Under suitable assumptions the dynamics of the normalized empirical densities $\Delta_{X_{t}}(\boldsymbol{p}, \boldsymbol{q})$ converges with $N \rightarrow \infty$ to a limiting dynamics of normalized continuum densities $f(\boldsymbol{p}, \boldsymbol{q} ; t)$.

In the following, we attach a superscript ${ }^{(N)}$ to $\mathbb{M}_{K}$ and $X \in \mathbb{M}_{K}$ to indicate that $\mathbb{M}_{K}=\mathbb{M}_{K}^{(N)}$ and $X=X^{(N)}$ change with $N$. We also write $H^{(N)}\left(X^{(N)}\right)$ for $H^{(N)}\left(\boldsymbol{p}_{1}, \ldots, \boldsymbol{q}_{N}\right)$, and similarly for the integrals $\boldsymbol{P}^{(N)}, \boldsymbol{C}^{(N)}$, and $\boldsymbol{J}^{(N)}$.

We are interested in a continuum limit in the sense that the particle positions $\boldsymbol{q}_{i}$ (we should write $Q_{i}$ ) converge in distribution as $N \rightarrow \infty$, i.e. (at time $t=0)$ we want $\int_{\mathbb{R}^{3}} \underline{\Delta}_{X_{0}^{(N)}}(\boldsymbol{p},.) \mathrm{d}^{3} p \stackrel{N \rightarrow \infty}{\longrightarrow} \rho(. ; 0) \in\left(\mathfrak{L}_{+, 1}^{1} \cap C_{b}^{0}\right)\left(\mathbb{R}^{3}\right)$ weakly (metriced by some Kantorovich-Rubinstein metric $d_{\mathrm{KR}}$ ). We are only interested in those $\rho$ for which $\iint V\left(\left|\boldsymbol{q}-\boldsymbol{q}^{\prime}\right|\right) \rho(\boldsymbol{q} ; t) \rho\left(\boldsymbol{q}^{\prime} ; t\right) \mathrm{d}^{3} q \mathrm{~d}^{3} q^{\prime}<\infty$, where $\iint$ is taken over $\mathbb{R}^{3} \times \mathbb{R}^{3}$. We also want the kinetic and potential energies to scale comparably with $N$ for large $N$. Since only $N$ summands contribute to the kinetic energy while the potential energy $\propto N^{2}$ (for $N \gg 1$ ) when $\int_{\mathbb{R}^{3}} \Delta_{X^{(N)}}(\boldsymbol{p}, \boldsymbol{q}) \mathrm{d}^{3} p \rightarrow \rho(\boldsymbol{q})$ in the stipulated sense (suppressing $t$ temporarily), to achieve equal $N$-scaling of kinetic and potential energies the rescaled particle momentum vectors $N^{-1 / 2} \boldsymbol{p}_{i}$ must converge in distribution, so that $N^{-2} \sum_{1 \leq i \leq N}\left|\boldsymbol{p}_{i}\right|^{2} \stackrel{N \rightarrow \infty}{\longrightarrow} 2 \varepsilon_{\text {kin. }}$. Thus we ask that $N^{3 / 2} \underline{\Delta}_{X^{(N)}}\left(N^{1 / 2} \boldsymbol{p}, \boldsymbol{q}\right) \stackrel{N \rightarrow \infty}{\longrightarrow}$ $f(\boldsymbol{p}, \boldsymbol{q}) \in\left(\mathfrak{L}_{+, 1}^{1} \cap C_{b}^{0}\right)\left(\mathbb{R}^{6}\right)$ weakly, such that $N^{-2} H^{(N)}\left(X^{(N)}\right) \stackrel{N \rightarrow \infty}{\longrightarrow} \mathcal{E}(f)=\varepsilon<$ $\infty$, where

$$
\begin{aligned}
\mathcal{E}(f)= & \frac{1}{2 m} \iint|\boldsymbol{p}|^{2} f(\boldsymbol{p}, \boldsymbol{q}) \mathrm{d}^{3} p \mathrm{~d}^{3} q \\
& +\frac{e^{2}}{2} \iiint \int V\left(\left|\boldsymbol{q}-\boldsymbol{q}^{\prime}\right|\right) f(\boldsymbol{p}, \boldsymbol{q}) f\left(\boldsymbol{p}^{\prime}, \boldsymbol{q}^{\prime}\right) \mathrm{d}^{3} p \mathrm{~d}^{3} q \mathrm{~d}^{3} p^{\prime} \mathrm{d}^{3} q^{\prime},
\end{aligned}
$$

is the "energy of $f . "$ It then follows also that $\boldsymbol{C}^{(N)}\left(X^{(N)}\right) \stackrel{N \rightarrow \infty}{\longrightarrow} \vec{Q}(f)=\mathbf{0}$, $N^{-3 / 2} \boldsymbol{P}^{(N)}\left(X^{(N)}\right) \stackrel{N \rightarrow \infty}{\longrightarrow} \overrightarrow{\mathcal{P}}(f)=\mathbf{0}$, and $N^{-3 / 2} \boldsymbol{J}^{(N)}\left(X^{(N)}\right) \stackrel{N \rightarrow \infty}{\longrightarrow} \overrightarrow{\mathcal{g}}(f)=\boldsymbol{l}$, where

$$
\begin{aligned}
& \vec{Q}(f)=\iint \boldsymbol{q} f(\boldsymbol{p}, \boldsymbol{q}) \mathrm{d}^{3} p \mathrm{~d}^{3} q \\
& \overrightarrow{\mathcal{P}}(f)=\iint \boldsymbol{p} f(\boldsymbol{p}, \boldsymbol{q}) \mathrm{d}^{3} p \mathrm{~d}^{3} q
\end{aligned}
$$

${ }^{5}$ The vanishing of $\vec{Q}(f)$ and $\vec{P}(f)$ follows of course from the fact that each $X^{(N)}$ is picked from a $\mathbb{M}_{K}^{(N)}$, so that necessarily $\boldsymbol{C}^{(N)}\left(X^{(N)}\right)=\mathbf{0}$ and $\boldsymbol{P}^{(N)}\left(X^{(N)}\right)=\mathbf{0}$ for all $N$. 


$$
\vec{\jmath}(f)=\iint \boldsymbol{q} \times \boldsymbol{p} f(\boldsymbol{p}, \boldsymbol{q}) \mathrm{d}^{3} p \mathrm{~d}^{3} q
$$

are the "center of mass _," "momentum _," and "angular momentum of $f$," respectively. The functionals $\overrightarrow{\mathfrak{g}}(f), \overrightarrow{\mathcal{P}}(f), \vec{Q}(f)$ are well-defined for $f \in \mathfrak{L}_{+, 1}^{1}$ whenever $\mathcal{E}(f)<\infty$ thanks to our hypotheses on $V$.

We are now ready to state our first dynamical mean-field limit theorem. It is understood throughout that $V$ in (18) satisfies the stipulated hypotheses. Recall that the list of invariants $\left\{I_{0}, \ldots, I_{9}\right\}$ is common to all Hamiltonian systems with a Hamiltonian of type (18).

Theorem 3. Let $H_{9}^{(N)}$ denote any Hamiltonian of type (18) for which the list of invariants $\left\{I_{0}, \ldots, I_{9}\right\}$ exhausts the invariants. Let

$$
\mathfrak{A}_{9}=\left\{f \in \mathfrak{L}_{+, 1}^{1}\left(\mathbb{R}^{6}\right): \mathcal{E}(f)<\infty,|\overrightarrow{\mathcal{P}}(f)|<\infty,|\overrightarrow{\mathcal{Q}}(f)|<\infty,|\overrightarrow{\mathcal{I}}(f)|<\infty\right\}
$$

denote the set of admissible densities on $\mathbb{R}^{6}$. Let $\left\{t \mapsto X_{t}^{(N)}\right\}_{N=2}^{\infty}$ denote a sequence of solutions of the Hamiltonian dynamics on $\mathbb{M}_{9}^{(N)}, N=2,3, \ldots$, generated by $H_{9}^{(N)}$. For the initial data, assume that $N^{3 / 2} \triangleq_{X_{0}^{(N)}}\left(N^{1 / 2} \boldsymbol{p}, \boldsymbol{q}\right) \stackrel{N \rightarrow \infty}{\longrightarrow}$ $f(\boldsymbol{p}, \boldsymbol{q} ; 0) \in \mathfrak{A}_{9}$ weakly. Then $N^{3 / 2} \underline{\Delta}_{X_{N^{-1 / 2}}^{(N)}}\left(N^{1 / 2} \boldsymbol{p}, \boldsymbol{q}\right) \stackrel{N \rightarrow \infty}{\longrightarrow} f(\boldsymbol{p}, \boldsymbol{q} ; t) \in \mathfrak{A}_{9}$ weakly, where $f(\boldsymbol{p}, \boldsymbol{q} ; t)$ is a weak solution to the Vlasov equation

$$
\partial_{t} f+m^{-1} \boldsymbol{p} \cdot \nabla f-\nabla\left(e^{2} V * \int f \mathrm{~d}^{3} p\right) \cdot \partial_{p} f=0
$$

with initial data $f(\boldsymbol{p}, \boldsymbol{q} ; 0)$. Moreover, $N^{-2} H^{(N)}\left(X_{N^{-1 / 2} t}^{(N)}\right) \rightarrow \mathcal{E}(f(. ; t))=\varepsilon$, $\boldsymbol{C}^{(N)}\left(X_{N^{-1 / 2} t}^{(N)}\right) \rightarrow \vec{Q}(f(. ; t))=\mathbf{0}$, and $N^{-3 / 2} \boldsymbol{P}^{(N)}\left(X_{N^{-1 / 2} t}^{(N)}\right) \rightarrow \overrightarrow{\mathcal{P}}(f(. ; t))=\mathbf{0}$, and $N^{-3 / 2} \boldsymbol{J}^{(N)}\left(X_{N^{-1 / 2} t}^{(N)}\right) \rightarrow \overrightarrow{\mathcal{g}}(f(. ; t))=\boldsymbol{l}$, with $\varepsilon$ and $\boldsymbol{l}$ independent of time.

Beside the integrals $I_{k}$ inherited from the isolating integrals $I_{k}$, the Vlasov dynamics conserves an infinitude of so-called Casimir functionals of $f$.

Theorem 4. Let $C: \overline{\mathbb{R}}_{+} \rightarrow \mathbb{R}$ be a continuous function for which $C[f(. ; 0)]=$ $\iint C(f(. ; 0)) \mathrm{d}^{3} p \mathrm{~d}^{3} q$ exists for $f \in \mathfrak{A}_{9}$. Let $t \mapsto f(. ; t)$ solve (25) with initial data $f(. ; 0)$. Then $\mathcal{C}[f(. ; 0)]=\mathcal{C}[f(. ; t)]$ for all $t$.

In particular, among the Casimir functionals of (25) are: the "normalization of $f$, '6

$$
\mathcal{N}(f)=\iint f(\boldsymbol{p}, \boldsymbol{q}) \mathrm{d}^{3} p \mathrm{~d}^{3} q,
$$

the "entropy of $f$,"

$$
\mathcal{S}(f)=-\iint f(\boldsymbol{p}, \boldsymbol{q}) \ln f(\boldsymbol{p}, \boldsymbol{q}) \mathrm{d}^{3} p \mathrm{~d}^{3} q .
$$

\footnotetext{
${ }^{6}$ Of course, $\mathcal{N}(f)=1$ for $f \in \mathfrak{A}_{9}$.
} 
If $H^{(N)}$ given in (18) is not of type $H_{9}^{(N)}$, then additional invariants $I_{k}$ $(k=10, \ldots, K>9)$ exist (because the list $\left(I_{1}, \ldots, I_{9}\right)$ is shared by all $H^{(N)}$ given by (18). The Lynden-Bells' Hamiltonian is of type $H_{10}^{(N)}$ for which the obvious generalization of Theorem 3 holds, while Theorem 4 continues to hold unchanged.

\subsection{The support of the stationary ensemble measures}

We begin by stating our mean-field limit theorem for $\underline{\mu}^{(N)}$; or rather: $\tilde{\mu}^{(N)}$.

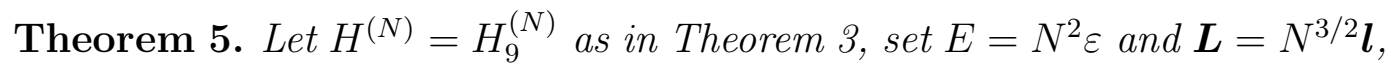
with $\varepsilon>0$ and $\boldsymbol{l}$ fixed, viable data. Let $\tilde{\mu}^{(N)}$ be given in (12) with $K=9$. Then

$$
\lim _{N \rightarrow \infty} \tilde{\mu}^{(N)}=\tilde{\mu}
$$

exists, and $\tilde{\mu}$ is a stationary measure for the adjoint dynamics inherited from $H^{(N)}$ in the limit $N \rightarrow \infty$. Furthermore, $\tilde{\mu}$ is supported on the set of maximizers of the entropy functional $\mathcal{S}(f)$, given in (27), taken from the set of trial densities

$$
\mathfrak{T}_{9}=\left\{f \in \mathfrak{L}_{+, 1}^{1}\left(\mathbb{R}^{6}\right): \mathcal{E}(f)=\varepsilon, \overrightarrow{\mathcal{P}}(f)=\mathbf{0}, \overrightarrow{\mathcal{Q}}(f)=\mathbf{0}, \overrightarrow{\mathcal{I}}(f)=\boldsymbol{l}\right\} .
$$

If $f_{\bullet}(\boldsymbol{p}, \boldsymbol{q})$ is a maximizer of $\mathcal{S}(f)$ over the set $\mathfrak{T}_{9}$, then $f_{\bullet}(\boldsymbol{p}, \boldsymbol{q})$ satisfies the fixed point (Euler-Lagrange) equation

$$
f_{\bullet}(\boldsymbol{p}, \boldsymbol{q})=e^{-1+\lambda_{\mathscr{C}}+\lambda_{\mathbb{E}}\left(\frac{1}{2 m}|\boldsymbol{p}|^{2}+e^{2}\left(V * \rho_{\bullet}\right)(\boldsymbol{q})\right)+\boldsymbol{\lambda}_{\jmath} \cdot \boldsymbol{q} \times \boldsymbol{p}+\boldsymbol{\lambda}_{P} \cdot \boldsymbol{p}+\boldsymbol{\lambda}_{Q} \cdot \boldsymbol{q}}
$$

where $\rho_{\bullet}=\int f_{\bullet} \mathrm{d}^{3} p$ and where $\lambda_{\mathscr{N}}, \lambda_{\mathscr{E}}, \boldsymbol{\lambda}_{\mathfrak{g}}, \boldsymbol{\lambda}_{\mathcal{P}}, \boldsymbol{\lambda}_{Q}$ are the Lagrange multipliers for the constraints $\mathcal{N}(f)=1, \mathcal{E}(f)=\varepsilon, \overrightarrow{\mathcal{I}}(f)=\boldsymbol{l}, \overrightarrow{\mathcal{P}}(f)=\mathbf{0}$, and $\vec{Q}(f)=\mathbf{0}$.

We are primarily concerned with the support of $\tilde{\mu}$. The interesting question of how $\tilde{\mu}$ varies over its support will be touched upon but briefly.

In addition to the maximizers of $\mathcal{S}(f)$ there may be other solutions of (30), yet only those solutions $f_{\bullet}$ for which $\mathcal{S}\left(f_{\bullet}\right)=\max$ over $\mathfrak{T}_{9}$ are in the support of $\tilde{\mu}$. All solutions are critical points for $\mathcal{S}(f)$. Independently of whether a solution of (30) maximizes $\mathcal{S}\left(f_{\bullet}\right)=\max$ over $\mathfrak{T}_{9}$ or not the following holds.

Theorem 6. Every solution of the fixed point equation (30) satisfying the constraints $\mathcal{N}\left(f_{\bullet}\right)=1, \overrightarrow{\mathcal{P}}\left(f_{\bullet}\right)=\mathbf{0}, \vec{Q}\left(f_{\bullet}\right)=\mathbf{0}$, and $\overrightarrow{\mathcal{I}}\left(f_{\bullet}\right)=\boldsymbol{l}$ factors into a product of a locally (at $\boldsymbol{q}$ ) shifted Maxwellian on $\boldsymbol{p}$ space and a purely space-dependent Boltzmann factor with $\boldsymbol{\lambda}_{P}=\mathbf{0}, \lambda_{\mathcal{E}}=-1 / k_{\mathrm{B}} T<0$, and 
$\boldsymbol{\lambda}_{\boldsymbol{y}}=\boldsymbol{\omega} / k_{\mathrm{B}} T$, where $T$ is the temperature and $\boldsymbol{\omega}$ the angular frequency vector of rotation of the dynamical system; thus

$$
f_{\bullet}(\boldsymbol{p}, \boldsymbol{q})=\left(\frac{1}{2 \pi m k_{\mathrm{B}} T}\right)^{\frac{3}{2}} \exp \left(-\frac{1}{2 m k_{\mathrm{B}} T}|\boldsymbol{p}-m \boldsymbol{\omega} \times \boldsymbol{q}|^{2}\right) \rho_{\bullet}(\boldsymbol{q})
$$

with $\rho_{\bullet}$ satisfying the fixed point equation on $\boldsymbol{q}$ space

$$
\rho_{\bullet}(\boldsymbol{q})=\frac{\exp \left(-\frac{1}{k_{\mathrm{B}} T}\left[e^{2}\left(V * \rho_{\bullet}\right)(\boldsymbol{q})-\frac{m}{2}|\boldsymbol{\omega} \times \boldsymbol{q}|^{2}\right]+\boldsymbol{\lambda}_{Q} \cdot \boldsymbol{q}\right)}{\int_{\mathbb{R}^{3}} \exp \left(-\frac{1}{k_{\mathrm{B}} T}\left[e^{2}\left(V * \rho_{\bullet}\right)\left(\boldsymbol{q}^{\prime}\right)-\frac{m}{2}\left|\boldsymbol{\omega} \times \boldsymbol{q}^{\prime}\right|^{2}\right]+\boldsymbol{\lambda}_{Q} \cdot \boldsymbol{q}^{\prime}\right) \mathrm{d} q^{\prime}}
$$

Here we also eliminated the Lagrange multiplier $\lambda_{\mathfrak{N}}$ using $\mathcal{N}\left(f_{\bullet}\right)=1$.

Theorems 5 and 6 hold for all Hamiltonian systems with Hamiltonian of type $H_{9}^{(N)}$, which covers - presumably - the majority of models with Hamiltonian given by (18) satisfying the hypotheses of Proposition $1 \mathrm{~b}$ together with those stipulated at the beginning of the section on $N \rightarrow \infty$. They are proven similarly as in [KiLe97]. It is straightforward to generalize Theorems 5 and 6 to the Hamiltonians given by (18) which are of type $H_{10}^{(N)}$, which includes the Lynden-Bells' Hamiltonian. In that case the entropy maximizer factors into a product of a locally (at $\boldsymbol{q}$ ) shifted Maxwellian on $\boldsymbol{p}$ space and a purely space-dependent Boltzmann factor. The shifted Maxwellian in (31) is known as a "rotating Maxwellian;" in the case of the Lynden-Bells' Hamiltonian we find a "rotating-dilating Maxwellian."

\section{Thermodynamics [sic!]}

By Theorem 6, w.r.t. Kantorovich-Rubinstein topology (metrizing weak convergence of measures) every solution of the fixed point equation (30) represents the microscopic density function on single particle phase space of an individual $N=\infty$ many particles system which locally at each $\boldsymbol{q}$ is in thermal equilibrium w.r.t. an instantaneously co-rotating frame at $\boldsymbol{q}$. A forteriori this is true also for the maximizers of $\mathcal{S}(f)$ over $\mathfrak{T}_{9}$. However, globally a solution to (30) - and therefore in particular any maximizer of $\mathcal{S}(f)$ over $\mathfrak{T}_{9}$ - may be a stationary solution of (25) but need not be. If a solution to (30) is a stationary solution of (25), then it describes a system which as a whole is either in a non-static stationary state or in a static state, depending on whether a non-vanishing velocity field $\overline{\boldsymbol{u}}(\boldsymbol{q})=m^{-1} \int \boldsymbol{p} f_{\bullet}(\boldsymbol{p}, \boldsymbol{q}) \mathrm{d}^{3} p$ exists or not, respectively. If a solution to (30) is not a stationary solution of (25), then it is necessarily a snapshot of a whole connected orbit of densities which solve the genuinely time-dependent Vlasov equation (25). Note though that all snapshot densities in such an orbit are necessarily maximizers of $\mathcal{S}(f)$ over $\mathfrak{T}_{9}$, for the Vlasov dynamics preserves 
the isolating integrals as well as normalization and entropy. All this will become more dramatically so for the rotating-dilating Maxwellians obtained for the Lynden-Bells' Hamiltonian of type $H_{10}^{(N)}$.

Incidentally, whenever these microstates of the system in the limit $N=$ $\infty$ vary sufficiently mildly, then they coincide with the mesostates and map bijectively into the macrostates. The upshot is: such solutions describe systems which in general are truly thermodynamical! This leads us to tentatively propose the following terminology.

The static maximizers of entropy $\mathcal{S}(f)$ over $\mathfrak{T}_{9}$ will be called thermostatic states. The non-static stationary entropy maximizers will be sanctioned thermostationary states; these include some (but not all) of those states which are frequently called "rotating equilibrium states." The non-stationary entropy maximizers are genuinely thermodynamical states. All this applies to the $N=\infty$ states.

The finite- $N$ meso- and macrostates for large enough $N$ are then wellapproximated by these $N=\infty$ states and can be categorized in these terms as well. This is the content of a law of large numbers as $N \rightarrow \infty$ over the empirical statistics over the particles in an individual system. Note that when there is a unique maximizer of entropy, then this law of large numbers and the one stated earlier for finite $N$ will give essentially the same results when $N$ is large enough, but only then.

\section{Closing remarks}

In this presentation I have tried to explain what equilibrium statistical mechanics is all about. My title "Statistical equilibrium dynamics" summarizes this as concisely as I could, in the following sense: Gibbs referred to Boltzmann's stationary ergodic ensembles as being in "statistical equilibrium" (so these two words are bound also in my title), but the "dynamics" in my title refers to the meso- or macrostate of the individual members of such an ensemble, which as we saw, may very well carry out a nontrivial large scale dynamics even in the limit $N \rightarrow \infty$. Yet, at any instant of time the individual macrostate of the $N=\infty$ system is a maximizer of the Boltzmann entropy function ( $-H$ function) subject to the the conservation laws and other scleronomic constraints.

In this presentation I have restricted myself to the general picture, though I mentioned specific systems without decending into the details. Those details will be given in a joint publication with Carlo Lancellotti [KiLa08].

Acknowledgment This work was supported in parts by the NSF under grants DMS-0103808 and DMS-0406951. Any opinions expressed in this paper are entirely those of the author and not those of the NSF. I thank Shelly Goldstein and Carlo Lancellotti for many valuable discussions. Finally, I thank 
the organizers, Alessandro Campa, Andrea Giansanti, Giovanna Morigi, and Francesco Sylos Labini, for the kind invitation to and the fantastic organization of this workshop, and for their generous help and support.

\section{References}

[Bal82] Balian, R., From microphysics to macrophysics, vol. I, Springer Verlag, New York (1982).

[Bol96] Boltzmann, L., Vorlesungen über Gastheorie, J.A. Barth, Leipzig (1896); English translation: "Lectures on Gas theory" (S.G. Brush, transl.), Univ. California Press, Berkeley (1964);

[Cha69] Chandrasekhar, S. Ellipsoidal figures of equilibrium, Yale Univ. Press, New Haven (1969); reprinted by Dover (1987).

[Dud02] Dudley, R.M., Real analysis and probability, Cambridge Univ. Press, Cambridge UK (2002).

[Dur96] Durrett, R., Probability: Theory and Examples, 2nd ed., Dunbury Press, Belmont (1996).

[Gib02] Gibbs, J.W., Elementary Principles in Statistical Mechanics, Yale Univ. Press, New Haven (1902); reprinted by Dover, New York (1960).

[Kie08] Kiessling, M. K.-H., "Misleading signposts along the de Broglie-Bohm road to quantum mechanics," Found. Phys. to appear (2008).

[KiLa08] Kiessling, M.K.-H., and Lancellotti, C., in preparation (2008).

[KiLe97] Kiessling, M.K.-H., and Lebowitz, J.L., "The microcanonical point vortex ensemble: Beyond equivalence," Lett. Math. Phys. 42:43-56 (1997).

[GoLe04] Goldstein, S., and Lebowitz, J. L., "On the (Boltzmann) entropy of nonequilibrium systems," Physica D 193:53-66 (2004).

[LBLB99] Lynden-Bell, D. and R.M., "Exact general solutions to extraordinary N-body problems," Proc. R. Soc. Lond. A 445:475-489 (1999).

[LBLB04] Lynden-Bell, D. and R.M., "Relaxation to a perpetually pulsating equilibrium," J. of Stat. Phys. 117:199-209 (2004).

[Pen70] Penrose, O., Foundations of Statistical Mechanics: A Deductive Treatment, Pergamon Press, Oxford (1970); reprinted by Dover (2005). 
[Rue69] Ruelle, D. Statistical Mechanics: Rigorous Results, Benjamin, New York (1969); reprinted in the "Advanced Book Classics" series of AddisonWesley, Reading (1989).

[Thi89] Thirring, W., Quantum Mathematical Physics, Springer, New York (1989). 